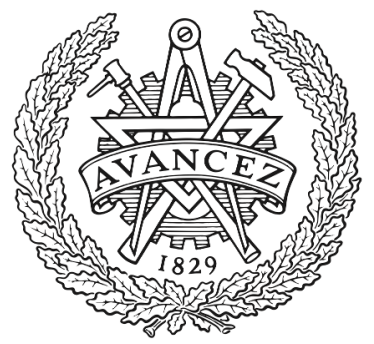

CHALMERS

UNIVERSITY OF TECHNOLOGY

\title{
Reducing Spiraling in Transducer Array Based Acoustic Levitation
}

Downloaded from: https://research.chalmers.se, 2023-04-26 03:49 UTC

Citation for the original published paper (version of record):

Andersson, C., Ahrens, J. (2020). Reducing Spiraling in Transducer Array Based Acoustic Levitation. IEEE International Ultrasonics Symposium, IUS, 2020-September.

http://dx.doi.org/10.1109/IUS46767.2020.9251489

N.B. When citing this work, cite the original published paper. 


\section{Reducing Spiraling in Transducer Array Based Acoustic Levitation}

\author{
Carl Andersson \\ Division of Applied Acoustics \\ Chalmers University of Technology \\ Gothenburg, Sweden \\ Email: carl.andersson@chalmers.se
}

\author{
Jens Ahrens \\ Division of Applied Acoustics \\ Chalmers University of Technology \\ Gothenburg, Sweden \\ Email: jens.ahrens@chalmers.se
}

\begin{abstract}
Ultrasonic transducer array based levitation can be applied for non-contact manipulation of small objects. The technique can sometimes suffer from instability issues in the form of uncontrolled oscillatory movement that builds up until the levitating object moves out of the levitation trap region and drops. We propose an additional condition to the established description of the levitation trap, and describe how the condition is used in numerical optimization of a cost function to obtain the phases and amplitudes of the elements in the array. The proposed condition successfully reduces the rotational components in the force field responsible for the uncontrolled increase in kinetic energy of the levitating object.
\end{abstract}

Index Terms-Radiation force, Acoustic Levitation, Ultrasound, Numerical Optimization

\section{Introduction}

Dynamic non-contact movement or containment of small objects is a useful application of acoustic levitation. For a fully dynamic system, the easiest way to create a reconfigurable sound field is to use a phased ultrasonic transducer array, see Figure 1. This is for example used as new human computer interaction interfaces where the dynamic capabilities of the system are used actively in response to user interaction $[1,2,3]$. Static applications also exist, e.g. containment of objects during analysis when the object cannot be levitated with optical or magnetic levitation due to size or material restrictions [4]. In dynamic levitation systems it happens frequently that the levitating object is not centered precisely in the middle of a levitation trap. It has been reported that levitating objects, in certain scenarios, start spinning in the trap and follow a spiraling path until they are ejected from the trap [5]. In most cases it is desirable that objects remain motionless in the trap and that when disturbed, intentionally or not, return as quickly and directly as possible to the center. If the spiraling behavior is reduced or removed entirely the overall stability of the system will be improved.

A powerful technique to find the phases and amplitudes of the elements in the array is to use numerical

This paper is part of a project that has received funding from the European Union's Horizon 2020 research and innovation programme under grant agreement No 737087.

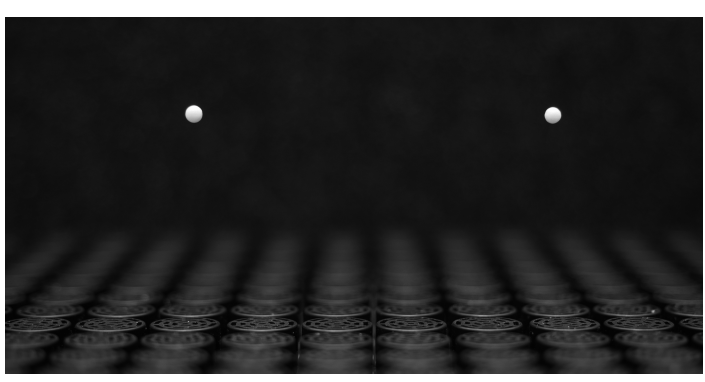

Fig. 1. Photograph of two polystyrene beads levitating above an ultrasonic transducer array as an example of how a levitating system can look.

optimization with a cost function derived from intuitive descriptions of what constitutes a trapping force $[6,7]$. The initial description of the trap conditions will influence the resulting force field in and around the trap. We propose an addition to the initial description of the trap conditions used in previous works to reduce the problem with objects spiraling in the force field. This additional part uses the curl of the force field as a descriptor of forces which accelerate object around the trap, and are responsible for the spiraling motion.

\section{Method}

\section{A. Trap Description}

An acoustic levitation trap is a region in space where an object, often a small lightweight spherical bead, remain stationary when placed there, i.e. the net force on the object is zero. For a levitation trap to be stable, there must be a restoring force to push the object back if it is disturbed from the center of the trap. This can be formulated mathematically as

$$
\begin{aligned}
F_{q}\left(\vec{x}_{0}\right) & =0 \\
\frac{\partial F_{q}}{\partial q}\left(\vec{x}_{0}\right) & <0
\end{aligned}
$$

where $F_{q}$ is the net force acting on the object in the Cartesian directions $q=x, y, z$, and $\vec{x}_{0}$ is the trap center. If these conditions are met, an object situated close to the trap center will always experience a force component 
directed towards the trap center. The conditions (1) and (2) do not, however, constrain force components perpendicular to the vector from the trap center to the object. A strong perpendicular force component will accelerate the object in a circular trajectory. As long as the force towards the center of the trap is sufficiently strong the object will eventually converge to the center of the trap, but it might do so in a spiraling path. If the perpendicular force is too strong it will accelerate the object to very high velocities, so that the constraining force will not suffice and the object is ejected from the trap.

This build-up of kinetic energy and momentum from the perpendicular force mean that the total energy of the object is not conserved, i.e., energy is continuously transferred from the field to the object. A field that enables such transfer of energy is rotational, i.e. $\nabla \times \vec{F} \neq 0$. To prevent this situation, we propose to additionally constrain the force field to have zero curl around the trap, as

$$
\nabla \times \vec{F}\left(\vec{x}_{0}\right)=0 .
$$

Since curl is composed of terms on the form

$$
\left(\frac{\partial F_{q}}{\partial w}-\frac{\partial F_{w}}{\partial q}\right) \hat{e}_{v}, \quad q \neq w \neq v,
$$

the condition (3) can be reformulated to state that the opposing partial derivative terms should be equal.

\section{B. Field Description}

In order to evaluate the trap conditions (1) to (3), a model for the radiation force is needed. In this work, we have chosen to work with small spherical objects so that the approximation $k a<1$ is valid, where $a$ is the radius of the spherical object and $k=\omega / c_{0}$ is the wavenumber of the impinging waves. In such cases there is a convenient formulation for the radiation force from arbitrary monofrequent sound fields, using sound field quantities in the center of the object [8], as

$$
\tilde{F}_{q}=\frac{\pi \kappa_{0}}{k^{5}} \Re\left\{A p \bar{p}_{q}+B\left(p_{x} \bar{p}_{x q}+p_{y} \bar{p}_{y q}+p_{z} \bar{p}_{z q}\right)\right\}
$$

where the subscripts on the sound pressure $p$ indicate partial derivatives with respect to the Cartesian coordinates, - indicates complex conjugation, $\kappa_{0}=1 /\left(\rho_{0} c_{0}^{2}\right)$ is the compressibility of the medium and

$$
\begin{aligned}
A & =-\frac{2 k^{2}}{9}\left(3(k a)^{3} f_{1}-\mathrm{i}(k a)^{6}\left(f_{1}^{2}+f_{1} f_{2}\right)\right) \\
B & =(k a)^{3} f_{2}+\mathrm{i} \frac{(k a)^{6}}{6} f_{2}^{2} \\
f_{1} & =1-\frac{\kappa}{\kappa_{0}}, \quad f_{2}=2 \frac{\rho-\rho_{0}}{2 \rho+\rho_{0}} .
\end{aligned}
$$

In these last expressions, $\rho$ and $\rho_{0}$ are the densities of the object and the medium, and $\kappa$ is the compressibility of the object. Note that the above expression refers only to the radiation force. The net force will typically also include a gravitational force $\vec{G}$, which is assumed constant in space and time.
The expression for the radiation force, (4), can be used to find explicit expressions for the force gradient terms involved in the conditions (2) and (3), as

$$
\begin{aligned}
\frac{\partial F_{q}}{\partial q}=\frac{\pi \kappa_{0}}{k^{5}} \Re\{ & A\left(p_{q} \bar{p}_{q}+p \bar{p}_{q q}\right)+B\left(p_{x q} \bar{p}_{x q}+p_{x} \bar{p}_{x q q}\right. \\
& \left.\left.+p_{y q} \bar{p}_{y q}+p_{y} \bar{p}_{y q q}+p_{z q} \bar{p}_{z q}+p_{z} \bar{p}_{z q q}\right)\right\}
\end{aligned}
$$

and

$$
\begin{aligned}
& \frac{\partial F_{q}}{\partial w}-\frac{\partial F_{w}}{\partial q}=\frac{2 \pi \kappa_{0}}{k^{5}}\left(\Im\{A\} \Im\left\{p_{q} \bar{p}_{w}\right\}\right. \\
&\left.+\Im\{B\} \Im\left\{p_{x q} \bar{p}_{x w}+p_{y q} \bar{p}_{y w}+p_{z q} \bar{p}_{z w}\right\}\right) .
\end{aligned}
$$

Since the sound field in this work is generated from a transducer array, the sound pressure and its derivatives can be calculated from the superposition of elementary source contributions. We model the transducer elements as points sources with a known directivity, i.e.

$$
p=\sum_{n} Q_{n} \frac{\mathrm{e}^{\mathrm{i} k r_{n}}}{r_{n}} D\left(\theta_{n}\right)
$$

where $Q_{n}=\alpha_{n} \mathrm{e}^{\mathrm{i} \varphi_{n}}$ is a complex value representing the amplitude and phase of the element, $r_{n}$ is the distance from the element to the object, $\theta_{n}$ is the angle between the transducer normal and the direction from the transducer to the object, and $D$ is a rotationally symmetric directivity function, typically that of a radiating circular piston or a circular ring. The calculus needed for the three orders of spatial derivatives of the pressure is somewhat lengthy, but in principle not particularly difficult. The final expressions are bulky, and will not be stated here.

\section{Numerical Optimization}

To find a combination of phases and amplitudes for the array elements to produce a sound field where the levitation conditions (1) to (3) are met, we use numerical optimization with the phases and amplitudes as the unknowns. A Broyden-Fletcher-Goldfarb-Shanno (BFGS) variant using limited memory and supporting bounded unknowns is used to minimize a cost function $[9,10]$. To improve the convergence rate and stability of the algorithm, the Jacobians of the levitation conditions w.r.t. the transducer phases and amplitudes are calculated analytically.

The levitation conditions cannot be optimized directly using this type of optimization, but needs to be converted to a single value cost function. Since two of the conditions, (1) and (3), are on the form $\vec{v}=0$, they can equivalently be expressed as $|\vec{v}|^{2}=0$, where the square is used to preserve cost function smoothness around the minimum. The last condition (2) is what gives us the restoring force in the trap. If the derivatives in said condition are larger in magnitude, the trap is stiffer and restores an object to its intended position faster. We can therefore choose the sum of the three derivatives as the single value quantifier for 
the trap stiffness. A similar technique has successfully been used in the past but without the condition of zero curl, and replacing the condition of zero force with a condition of zero pressure in the trap center, i.e. $\left|p\left(\vec{x}_{0}\right)\right|^{2}=0[6]$.

In total we have the cost function

$$
O=w_{f}|\vec{F}|^{2}+w_{s}(\nabla \cdot \vec{F})+w_{c}|\nabla \times \vec{F}|^{2}
$$

where all field quantities are evaluated in the center of the trap. Since the magnitude of the conditions are on very different scales they have been weighted appropriately with weights $w_{f}, w_{s}, w_{c}$. In the specific case tested here, weights $w_{f}=10^{12}, w_{s}=1$, and $w_{c}=10^{6}$ gave reasonable results.

\section{Results}

To fully show the difference between an optimization result with no curl minimization and a result where the proposed curl minimization is used, the simulated scenario is chosen so that the previously used method converges to a result where there is notable curl in the force field. We simulate a 16x16 element single-sided flat transducer array, i.e. all array elements are in the same plane. The transducer elements are modeled as point sources operating at $40 \mathrm{kHz}$ in air, with a directivity function corresponding to a circular ring radiator with a radius of $2.9 \mathrm{~mm}$, which was the simple analytical model that best fit to our directivity measurements [11]. The elements are spaced in a square grid, $10 \mathrm{~mm}$ apart, which corresponds to approximately $1.17 \lambda$, indicating that spatial aliasing might influence the final sound field. The trap center is chosen to be $z=60 \mathrm{~mm}$ above the array, $x=30 \mathrm{~mm}$ off center in one axis, and $y=4 \mathrm{~mm}$ off center along the other axis. The spherical objects in the simulations have a radius of $a=1 \mathrm{~mm}$, density $\rho=25 \mathrm{~kg} / \mathrm{m}^{3}$, and speed of sound $c=2350 \mathrm{~m} / \mathrm{s}$.

Figure 2 shows slices of the net force acting on the levitating object for different positions in the horizontal $(x y)$ and one vertical $(x z)$ plane around the trap, with and without the proposed method for curl reduction. It is clear that the proposed method reduces the curl in the field as intended, while still keeping the converging parts of the field. In the horizontal slice, the force field without curl reduction shows a strong circular component, pushing the object around the trap. The field obtained from the proposed method instead has two strong force lobes along one axis, and weaker but still converging forces along the other axis.

The force fields in the vertical slice both show convergence towards a tilted line in the $x z$-plane. This is related to the position of the trap in comparison to the array center. The field without reduction of curl creates a strong upwards force outside this central line, while the force field where the curl is reduced pushes the object directly towards the central line. Considering an object that is not in the center of the trap, it seems that the force field from

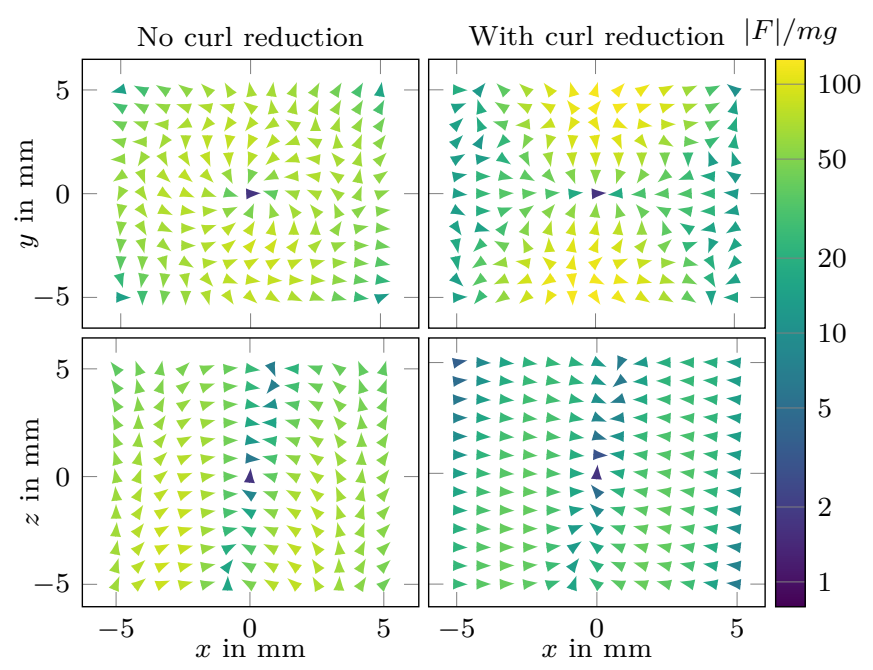

Fig. 2. Force fields at different positions of the spherical object in slices around the trap, after optimization of the cost function (5) excluding curl reduction (left, $w_{c}=0$ ) and including curl reduction (right, $\left.w_{c}>0\right)$. The slices are through the trap position at $(0,0$, 0 ), in the horizontal plane ( $x y$, top) parallel to the array, and in the vertical plane ( $x z$, bottom) normal to the array. The forces are shown as factors of the gravitational force on the levitated object, on a logarithmic scale.

the proposed method will cause the object to take a more direct path to the center.

Figure 3 shows simulated trajectories for an object placed at a small $(\sim 1 \mathrm{~mm})$ offset from the trap center in the two force fields. The field produced with the proposed method creates a trap in which the object settles after an initial oscillatory phase. This oscillation is expected since the trap is not very damped, including a drag force as the only damping [12]. It is clear that the oscillation frequency in the $z$-direction is lower than in the $y$-direction, and that there is a correlation between the movement along the $z$-axis and the $x$-axis. The difference in frequency is related to the difference in trapping strength or stiffness, since the resonance frequency of an undamped oscillator is $\omega=\sqrt{s / m}$ with $s$ as the stiffness and $m$ as the mass. Figure 2 clearly shows how the horizontal force, and therefore stiffness, is much stronger than the vertical force. The correlation between $x$ and $z$ can again be understood from Figure 2, where the trap center moves towards larger $x$ for larger $z$. This is a consequence of the chosen trap position, and will change accordingly if the position is changed.

An object placed in the trap created without curl minimization will be ejected almost instantaneously, as shown with the dashed lines in Figure 3. For the scenario used here, even an object placed in the middle of the trap with strong curl is ejected very quickly, while an object placed in the center of the trap with reduced curl stays precisely were placed. 

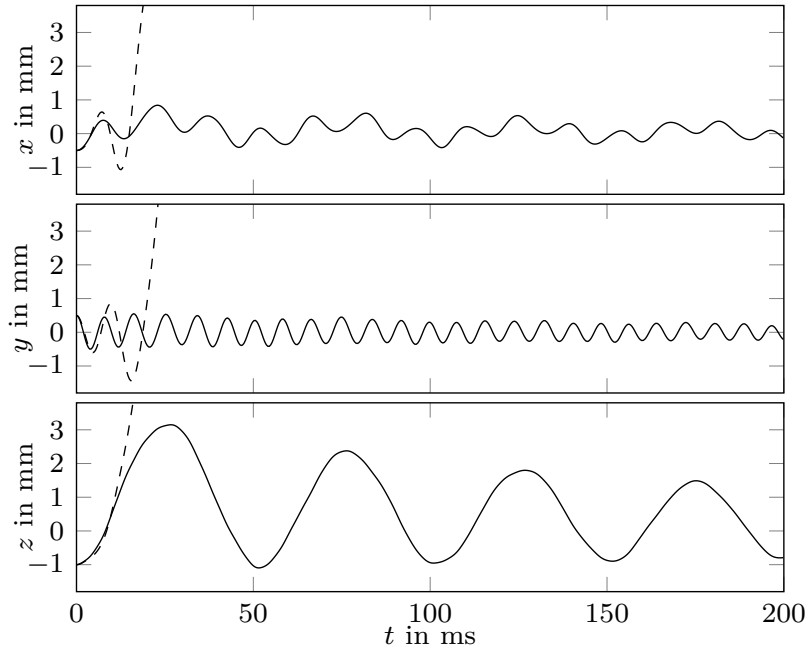

Fig. 3. Simulations of the trajectory of objects with an initial offset in the traps created by the two methods. The solid lines are from a simulation using the proposed curl reduction method, while the dashed lines are without it.

\section{Discussion}

The proposed method can reduce the curl in the force field acting on small levitating spherical objects when the sound field is created using transducer arrays. The benefit of reducing the curl of the force field is not proven, but easily understood intuitively and shown for at least one simulated case. A spherical object that happens to be offcenter in the trap from a force field with a strong curl will not accelerate directly towards the center of the trap, but rather in a perpendicular trajectory. Since the force field is not conservative, the total energy of the object might increase to the point where it can escape the potential well described by the restoring force component in the field. Reducing or eliminating the curl in the force field should eliminate or reduce the possibility of such energy build-up, and create a more stable trap.

If the array is used to move an object in a certain path, the object is intentionally off-center in the trap since this is what causes the desired movement. Designing a movement scheme when the object always moves directly towards the trap is relatively easy: move the trap in the desired direction of movement. If the object will move perpendicularly to the vector from the object to the trap it will be much more difficult to create fast and smooth movement.

It is also of interest to note that it is not enough to consider only the force field to see if an object will be trapped or not. The force field shown to the left in Figure 2 is converging towards the center. An object placed in the field will, however, not converge to the center.

\section{References}

[1] E Freeman et al. "Levitating Object Displays with Interactive Voxels". In: th ACM International Symposium on Pervasive Displays (2018).

[2] Myroslav Bachynskyi, Viktorija Paneva, and Jörg Müller. "LeviCursor". In: the 2018 ACM International Conference. New York, New York, USA: ACM Press, 2018, pp. 253-262.

[3] Euan Freeman et al. Point-and-Shake: Selecting from Levitating Object Displays. Selecting from Levitating Object Displays. New York, New York, USA: ACM, Apr. 2018.

[4] Ljiljana Puskar et al. "Raman acoustic levitation spectroscopy of red blood cells and Plasmodium falciparum trophozoites". In: Lab on a Chip 7.9 (2007), pp. 1125-1131.

[5] Asier Marzo, Mihai Caleap, and Bruce W Drinkwater. "Acoustic Virtual Vortices with Tunable Orbital Angular Momentum for Trapping of Mie Particles". In: Physical Review Letters 120.4 (Jan. 2018), p. 044301.

[6] Asier Marzo et al. "Holographic acoustic elements for manipulation of levitated objects". In: Nature Communications 6.1 (2015), p. 4316.

[7] Carl Andersson and Jens Ahrens. "A Method for Simultaneous Creation of an Acoustic Trap and a Quiet Zone". In: 2018 IEEE 10th Sensor Array and Multichannel Signal Processing Workshop (SAM). IEEE, 2018, pp. 622-626.

[8] Oleg A Sapozhnikov and Michael R Bailey. "Radiation force of an arbitrary acoustic beam on an elastic sphere in a fluid". In: Journal of the Acoustical Society of America 133.2 (Feb. 2013), pp. 661-676.

[9] Pauli Virtanen et al. "SciPy 1.0: Fundamental Algorithms for Scientific Computing in Python". In: Nature Methods (Feb. 3, 2020), pp. 1-12. issn: 15487105. doi: 10.1038/s41592-019-0686-2.

[10] Ciyou Zhu et al. "Algorithm 778: L-BFGS-B: Fortran subroutines for large-scale bound-constrained optimization". In: ACM Transactions on Mathematical Software (TOMS) 23.4 (Dec. 1997), pp. 550-560.

[11] Carl Andersson and Jens Ahrens. Database of Ultrasonic Transducer Radiation Characteristics. Dec. 2017. doi: 10.5281/zenodo.1118386. url: https://doi. org/10.5281/zenodo.1118386.

[12] Phillip P Brown and Desmond F Lawler. "Sphere Drag and Settling Velocity Revisited". In: Journal of Environmental Engineering 129.3 (Mar. 2003), pp. 222-231. 